\title{
EFECTOS A LARGO PLAZO DE UN PROGRAMA DE INTERVENCIÓN COGNITIVO CONDUCTUAL EN LA SINTOMATOLOGÍA MENOPÁUSICA: RESULTADOS DE UN ESTUDIO PILOTO
}

\author{
SONIA GUTIÉRREZ y CRISTINA LARROY \\ Facultad de Psicología, Universidad Complutense de Madrid
}

\begin{abstract}
Resumen: La menopausia se asocia a una importante variedad de síntomas físicos, psicológicos y sociales, susceptibles de ser tratados mediante técnicas cognitivo-conductuales. En este trabajo, 28 mujeres recibieron una intervención grupal de ocho sesiones semanales de dos horas para controlar depresión, ansiedad y otros síntomas asociados. Se realizó una medida postratamiento y un seguimiento a los 6 meses. Los resultados muestran una reducción significativa de la mayoría de la sintomatología asociada (incluyendo depresión y ansiedad), tanto respecto al periodo de línea base como al grupo control, y tanto en postratamiento como en seguimiento (obteniéndose reducciones mayores en este último periodo para algunas variables). Estos últimos resultados son los que aquí se analizan. La relevancia del trabajo radica, precisamente, en que nunca antes se habían obtenido datos de un seguimiento tan prolongado.

Palabras clave: Menopausia, intervención psicológica, efectos a medio / largo plazo, estudio piloto.

Long term effects of a cognitive-behavioral intervention in menopausal symptomatology: A pilot study

Abstract: Menopause is related to an important variety of physical, psychological and social symptoms, susceptible to be treated by means of cognitive-behavioral techniques. Twenty-eight women participated in a group intervention of eight two-hour weekly sessions directed at reducing depression, anxiety and related symptoms. Results were measured at end of treatment and at 6 months. The results show a significant reduction of most of the associated symptoms (including depression and anxiety) compared to base line as well as to the control group, and both in posttreatment and in follow up (being greater at this time than in post-treatment, for some symptoms). These last results are analysed. No other study offers a follow up as prolonged.
\end{abstract}

Keywords: Menopause, psychological intervention, medium/ long-term effects.

\section{INTRODUCCIÓN}

Se conoce con el término "menopausia" al cese permanente de las menstruaciones debido al agotamiento de la actividad folicular ovárica que forma parte del proceso natural de envejecimiento de la mujer.

La menopausia suele aparecer entre los 45 y los 55 años. En España, la media de inicio se sitúa en torno a los 51,4 años (SEGO, 2006),

Recibido: 20-abril-2009; aceptado: 30-junio-2009.

Correspondencia: Cristina Larroy García, Facultad de Psicología, Universidad Complutense de Madrid, Campus de Somosaguas s/n, 28223 Madrid. Correo-e: clarroy@psi.ucm.es. siendo considerada precoz cuando aparece antes de los 45 años. En nuestro país, la menopausia sobreviene de forma natural a cerca de 270.000 mujeres cada año, lo que implica que alrededor del $20 \%$ de la población femenina española se encuentra en periodo menopáusico.

A pesar de esta elevada incidencia (4.5 millones de españolas), el desconocimiento sobre la menopausia, su sintomatología, causas y efectos, es aún muy importante; de hecho, el $61 \%$ de las mujeres que se encuentra en esta etapa vital desconoce que determinados síntomas, como la osteoporosis, los trastornos vasculares o las alteraciones del estado de ánimo son producto de la menopausia (SEGO, 2006). 
Debe considerarse que la menopausia acontece en un momento determinado de la vida de la mujer en el que confluyen otros cambios vitales importantes, sociales y familiares, que también han de tenerse en cuenta (pérdida de la juventud $\mathrm{y}$ del atractivo físico, cuidado de personas mayores, cuidado de nietos, jubilación, problemas sexuales, etc.), pues pueden provocar una valoración negativa de la experiencia de la menopausia. Además, el término "menopausia" ha sido utilizado por la sociedad en multitud de ocasiones en forma despectiva o como sinónimo de enfermedad o trastorno, por lo que su aparición puede llegar a convertirse en un suceso verdaderamente estresante para algunas mujeres. Sin embargo, la menopausia no es un trastorno en sí mismo sino una etapa de la vida de la mujer, aunque debido al cese de la actividad hormonal pueden producirse una serie de alteraciones físicas y psicológicas.

La intensidad y variedad de la sintomatología parece estar mediada por diferencias culturales (Matthews, 1991). Así, en aquellas culturas donde la mujer madura es considerada importante por su experiencia y se encuentra integrada dentro de su estatus social, apenas refiere sintomatología relacionada con la menopausia. Por el contrario, en las civilizaciones occidentales, donde la belleza y la juventud son excesivamente valorados por la sociedad, o en aquellas culturas donde el papel reproductivo es fundamental, la mujer madura atraviesa el periodo climatérico con muchas más dificultades y con una sintomatología más intensa (Anderson, Yoshizawa, Gollschewski, Atogami, y Courtney, 2004; Fu, Anderson y Courtney,
2003; Pelcastre-Villafuerte, Garrido-Latorre. y de León-Reyes, 2001).

La sintomatología de la menopausia puede ser muy variada, aunque existen algunos síntomas que se repiten con frecuencia. Estos síntomas están recogidos en la Tabla 1.

La intervención tradicional en la menopausia es de carácter médico: la terapia hormonal sustitutiva (THS). Sin embargo, la imposibilidad de aplicarla a todas las mujeres que sufren sintomatología, por sus contraindicaciones y por sus efectos secundarios, ha propiciado la búsqueda de nuevos tipos de intervenciones. Además, la THS no es eficaz en la remisión de la sintomatología ansiosa y depresiva en mujeres menopáusicas, por lo que se hace necesaria la intervención desde una perspectiva multidisciplinar. El abordaje de la menopausia desde la perspectiva cognitivo-conductual es relativamente reciente por lo que los trabajos son escasos, pero pueden citarse algunos, como los de Camuñas, García, Vivas, Morales, Aranda, y Cano, A. (2001); Hunter y Liao (1996), Sueiro, Carbulleira, Perdiz, Rodríguez y González (1999); o los de Larroy (2004), Larroy, Gutiérrez y León (2004), o Larroy y Gutiérrez (2009) que abarcan la sintomatología menopáusica y la mejora de la calidad de vida de las pacientes. Todos estos estudios hallan mejoras (totales o parciales) en la sintomatología menopáusica, pero sus resultados no abarcan más allá que el postratamiento, sin que, hasta el momento, se hayan estudiado los efectos de las intervenciones a largo plazo. Sin embargo, los estudios que evalúan los efectos de la intervención en los trastornos abordados por la Psicología de la

Tabla 1. Sintomatología asociada a la menopausia

\begin{tabular}{ccc}
\hline Síntomas vegetativos & Síntomas metabólicos & Síntomas Psíquicos \\
\hline Sofocos & Osteoporosis & Irritabilidad \\
Sudoración & Ateromatosis & Ansiedad \\
Palpitaciones & Atrofia cutánea & Depresión \\
Cefaleas & Atrofia urogenital & Nerviosismo \\
Vértigo & Artralgias & Insomnio \\
Opresión precordial & Mialgias & Disminución líbido \\
Zumbido de oídos & Obesidad & Pérdida de memoria \\
Hipertensión arterial & & Melancolía \\
& & Fatiga mental \\
\hline
\end{tabular}


Salud son frecuentes y sumamente útiles desde la perspectiva clínica: conocer cómo evolucionan los síntomas después de la intervención, si la mejoría se mantiene a lo largo del tiempo, y si los cambios difieren, también a largo plazo, de los experimentados por el grupo control, es fundamental para establecer la eficacia real de la intervención. Este es, precisamente, el objetivo del presente trabajo: analizar los resultados a largo plazo sobre la sintomatología menopáusica de una intervención cognitivo-conductual, que ha demostrado su eficacia a corto plazo. Se retoma para ello el trabajo de Larroy y Gutiérrez (2009). Este último programa, de carácter netamente cognitivo-conductual, se desarrolló en ocho sesiones semanales, de dos horas, con un formato grupal. Se elige este formato por sus múltiples ventajas: ha demostrado ser tan eficaz como la intervención individual en este tipo de trastornos (Philips, 1991; Larroy, 2004), las componentes del grupo se prestan apoyo mutuo, favoreciendo la adhesión a la intervención y el cumplimiento de las tareas, y se abaratan los costes, incrementándose la eficiencia. Los resultados obtenidos en este estudio en el postratamiento indicaron una remisión significativa de la sintomatología física y psicológica de la menopausia y una mejoría significativa de la calidad de vida en el grupo experimental (no así en el control, que no experimentó cambios significativos). Es destacable el hecho de que el estudio que aquí se presenta sea el primer trabajo de intervención cognitivo-conductual que ofrece datos de evaluación de seguimiento a largo plazo (seis meses).

\section{MÉTODO}

\section{Participantes}

Se contactó con las Concejalías y Casas de la Mujer de diferentes ayuntamientos de la Comunidad de Madrid donde se realizaban actividades de ayuda a la mujer, con el fin de ofrecer el programa. En estos centros se invitó a que las mujeres con sintomatología menopáusica participaran en la presente investigación. En la fase de intervención, el grupo experimental estuvo compuesto por 28 mujeres. Su media de edad era de 48,86 años y el rango oscilaba entre los 42 y 56 años $(\mathrm{DT}=3,61)$. El grupo control estaba compuesto por 25 mujeres, todas ellas cumplían los criterios de inclusión. La media de edad era de 49,88 años y el rango oscilaba entre los 44 y 55 años $(\mathrm{DT}=2,65)$. Tras el periodo de seguimiento, las muestras de ambos grupos (experimental y control) se redujeron por diferentes causas (incompatibilidades horarias con la sesión de evaluación, problemas familiares y cambios de domicilio) quedando finalmente 9 mujeres en el grupo experimental y 11 mujeres en el grupo control.

En la Tabla 2 se describen las variables sociodemográficas de la muestra.

\section{Instrumentos de evaluación}

Para evaluar las variables dependientes (sintomatología asociada a la menopausia y calidad de vida) se utilizaron los siguientes instrumentos:

Índice Menopáusico de Kupperman y Blatt (BMI, Blatt's menopausal index; Kupperman y Blatt, 1953). El BMI está constituido por 11 síntomas, evaluados mediante una escala de intensidad de 0 a 3 , donde 0 índica ausencia del síntoma y 3 intensidad máxima en el mismo. El índice menopáusico se obtiene multiplicando un factor, dado por los autores, por el índice de intensidad indicado por las mujeres. A aquellos síntomas más característicos de la menopausia (vasomotores) se les asigna un factor más elevado. El rango de puntuación oscila de 0 a 48 . Un índice por debajo de 15 se considera ligero, entre 15 y 20 moderado, entre 20 y 35 de moderado a alto y por encima de 35 , grave. El BMI es uno de los instrumentos más utilizados para la evaluación de los síntomas de la menopausia, hasta el punto de haberse constituido en referencia obligada para la mayoría de los investigadores de la menopausia (De la GándaraMartín, 1997). Los puntos de corte indicados son los se utilizan actualmente en España (Magnani, et al., 1999).

Escala hospitalaria de ansiedad y depresión (HADS, Hospital Anxiety and Depression Scale; Zigmond y Snaith, 1983). Debido a que el BMI ha recibido algunas críticas acerca de la 
Tabla 2. Variables sociodemográficas de la muestra en el pretratamiento y seguimiento

\begin{tabular}{|c|c|c|c|c|c|c|c|c|}
\hline \multirow[b]{2}{*}{ Variables } & \multicolumn{2}{|c|}{$\begin{array}{l}\text { Grupo clínico } \\
\text { experimental } \\
\quad(n=28)\end{array}$} & \multicolumn{2}{|c|}{$\begin{array}{l}\text { Grupo control } \\
\qquad(n=25)\end{array}$} & \multicolumn{2}{|c|}{$\begin{array}{l}\text { Grupo clínico } \\
\text { experimental } \\
\text { seguimiento } \\
\quad(n=9)\end{array}$} & \multicolumn{2}{|c|}{$\begin{array}{l}\text { Grupo Control } \\
\text { seguimiento } \\
\quad(n=11)\end{array}$} \\
\hline & $\%$ & Media & $\%$ & Media & $\%$ & Media & $\%$ & Media \\
\hline Edad & & 48,86 & & 49,88 & & 50,44 & & 51 \\
\hline \multicolumn{9}{|l|}{ Estado civil } \\
\hline Casada & $89,3 \%$ & & $76 \%$ & & $100 \%$ & & $73 \%$ & \\
\hline Separada & $10,7 \%$ & & $24 \%$ & & $0 \%$ & & $27 \%$ & \\
\hline \multicolumn{9}{|l|}{ Situación laboral } \\
\hline Activa & $10,7 \%$ & & $28 \%$ & & $11 \%$ & & $9 \%$ & \\
\hline Ama de casa & $85,7 \%$ & & $72 \%$ & & $78 \%$ & & $91 \%$ & \\
\hline Parada & $3,6 \%$ & & & & $11 \%$ & & & \\
\hline N..$^{o}$ de hijos & & 2,32 & & 2,20 & & 2 & & 2,55 \\
\hline \multicolumn{9}{|l|}{$\begin{array}{l}\text { Población de origen/ centro impartición } \\
\text { del programa }\end{array}$} \\
\hline Concejalía Mujer Leganés & $42,9 \%$ & & & & $78 \%$ & & & \\
\hline Casa de Mujer Fuenlabrada & $50 \%$ & & & & $0 \%$ & & & \\
\hline Concejalía Mujer de Collado-Villalba & $7,1 \%$ & & & & $22 \%$ & & & \\
\hline Leganés & & & $36 \%$ & & & & $36 \%$ & \\
\hline Fuenlabrada & & & $32 \%$ & & & & $27 \%$ & \\
\hline Móstoles & & & $12 \%$ & & & & $9 \%$ & \\
\hline Alcorcón & & & $12 \%$ & & & & $9 \%$ & \\
\hline Collado-Villalba & & & $8 \%$ & & & & $18 \%$ & \\
\hline
\end{tabular}

insuficiente evaluación de los síntomas psicológicos (Salvatierra, 1992), se ha considerado oportuno evaluar éstos mediante otro instrumento: el HADS. Las razones de su elección son: es utilizable en el ámbito comunitario, es fácil de cumplimentar y suministra una clara distinción entre los conceptos de ansiedad y depresión (De las Cuevas, García-Estrada y González, 1995). Este instrumento ha sido traducido a la mayoría de las lenguas, entre ellas la española, siendo estas traducciones fiables y validadas en numerosos estudios (Moorey et al., 1991). La escala posee una buena especificidad y sensibilidad en la detección de la ansiedad y depresión en pacientes físicamente enfermos (Barczak, Kane, y Andrews; 1988; Zigmond y Snaith, 1983). Consta de dos series de siete ítems, correspondientes a las subescalas de ansiedad y depresión. Cada ítem es valorado de 0 a 3 puntos, según su intensidad. Las puntuacio- nes mayores de 10 en cada subescala se consideran indicativas de morbilidad. Una puntuación de 8 a 10 se interpreta como moderada y las puntuaciones inferiores a 8 indican ausencia de morbilidad.

Cuestionario de valoración de calidad de vida, en mujeres de 45 a 64 años (Sánchez-Cánovas, 1999). Consta de 22 ítems, evaluados mediante una escala tipo Likert de 1 a 5, en función del grado de acuerdo con el ítem. La escala recoge información de contenidos relacionados con el climaterio, ansiedad, depresión, sexualidad, apoyo social y familiar. El cuestionario ofrece una puntuación global de la calidad de vida mediante la suma de las puntuaciones parciales de todos los contenidos relacionados con el climaterio. Se eligió este instrumento por tratarse del único cuestionario de calidad de vida específico para el climaterio que, en nuestro país, cumple los requisitos psicométricos exigibles (y porque la 
mejora en la calidad de vida es uno de los objetivos del estudio).

\section{Procedimiento}

Se utilizó un diseño cuasi-experimental con medidas repetidas y grupo control, siendo la variable independiente la intervención y las variables dependientes la sintomatología asociada a la menopausia y la calidad de vida. Dado que el programa se ofertó a todas las mujeres que acudían a las diferentes Casas de la Mujer fue necesaria la evaluación previa de la sintomatología para detectar qué mujeres presentaban sintomatología clínica. Se establecieron como criterios de inclusión en el grupo clínico: obtener una puntuación igual o superior a 8 en las escalas de ansiedad y/o depresión del HADS y una puntuación igual o superior a 20 en el BMI. Ambos límites en las puntuaciones de estos inventarios indican presencia de sintomatología moderada o elevada. Las mujeres que cumplían estos criterios se asignaban al grupo experimental. Las mujeres que no alcanzaban estos criterios también recibieron tratamiento pero los resultados no se tuvieron en cuenta para este estudio Se consideró criterio excluyente el estar o haber estado recientemente sometido a una terapia hormonal, dado que podía falsear los resultados del estudio.

Por cuestiones éticas y por depender de instituciones públicas, se decidió la admisión previa al programa de intervención a todas aquellas mujeres que lo demandaran en los centros en los que se llevara a cabo ésta y que no cumplieran los criterios de exclusión establecidos. Para hacer más homogéneos los grupos, la muestra del grupo control se reclutó en las mismas localidades y en localidades cercanas a éstas, a través de la técnica de "la bola de nieve": en primer lugar se contactó con unas mujeres que acudían a los centros (pero no al taller) y gracias a éstas se fue obteniendo más muestra. Es su caso, se les informó de que sólo debían responder a una serie de preguntas acerca de su estado actual relacionado con su etapa climatérica en diferentes momentos. Al igual que con el grupo experimental, sólo se tuvieron en cuenta para el estudio los resultados de aquellas mujeres con puntuaciones indicativas de sintomatología clínica en línea base pre-tratamiento.

El programa de intervención fue llevado a cabo por las psicólogas alumnas del Máster en Psicología Clínica y de la Salud de la Universidad Complutense de Madrid (UCM). Por razones externas a la investigación, se realizaron parejas de terapeutas para cada uno de los centros. Se midió la posible interferencia de esta variable, por no poder aplicarse la constancia o balanceo como método de control, comprobándose que era nula.

Se diseñó un programa de ocho sesiones de dos horas de duración, de periodicidad semanal y de carácter grupal y participativo, basado en el procedimiento de Larroy, Gutiérrez y León (2004). El programa de intervención constaba de los siguientes componentes: psicoeducación en menopausia; técnicas de desactivación; ejercicios de Kegel y reeducación sexual; identificación y control de preocupaciones excesivas y creencias irracionales; entrenamiento en solución de problemas y en administración del tiempo. El programa se desarrolló según se muestra en la Tabla 3. Una información más detallada puede encontrarse en Larroy y Gutiérrez (2009).

1. Educación del proceso de la menopausia. Se incluye porque la información es un factor primordial en cualquier programa de intervención (Magnani et al., 1999; Marín, Sánchez-Cánovas, Dupuy, 1995; Sueiro et al.,1999;), y más en la menopausia, por tratarse de un tema relativamente tabú, desconocido y centro de mitos o falsas creencias en diferentes sociedades y culturas (Marín, 1996; SEGO, 2006).

2. Relajación muscular progresiva y respiración diafragmática. Diferentes trabajos (Camuñas et al., 2001; Freedman y Woodward, 1992; Germaine y Freedman,1984; Hunter, 2003; Hunter y Liao, 1996; Keefer, 2003; Stevenson y Delprato, 1983; Sueiro et al., 1999) han utilizado las técnicas de relajación y respiración para el alivio de la sintomatología menopáusica. La relajación y la respiración consiguen la desactivación del sistema nervioso autónomo y, por tanto, la reducción de la ansiedad y el estrés. Diferentes trabajos han encontrado una relación entre el estrés 
y la aparición de sofocos. Ambas técnicas se han utilizado especialmente para la reducción de los síntomas vasomotores (sofocos y sudores nocturnos), el insomnio y la ansiedad, aunque también ayudan al alivio de la sintomatología en general, contribuyendo a mejorar la valoración subjetiva de la experiencia menopáusica.

3. Promoción de la salud, mediante aspectos educativos en ejercicio y nutrición. El objetivo de esta estrategia es destacar la importancia de unos hábitos de saludables para la prevención de la aparición de trastornos tales como la osteoporosis y trastornos cardiovasculares (OMS, 1996; Palacios, Menéndez, Jurado, y Castaño, 1998; Rosario, Villani, Harris y Klein (2003).

4. Entrenamiento en ejercicios de Kegel y opciones para combatir la sequedad vaginal. Se hace necesario abordar los trastornos de la musculatura del suelo pélvico en la perimenopausia, promoviendo técnicas de intervención para la prevención y el tratamiento de éstos, así como de los problemas sexuales derivados. Los más utilizados son los "ejercicios de Kegel" utilizados en diferentes programas para fortalecer el suelo pélvico (Magnani et al., 1999; Fernández, Magnani, Fernández, y Fernández-Arcilla, 2000).

5. Identificación y control de preocupaciones excesivas y creencias irracionales. Durante la menopausia pueden darse en las mujeres una serie de creencias irracionales o preocupaciones excesivas (Sueiro et al. 1999), representando éstas un papel muy importante en el desarrollo de alteraciones emocionales (ansiedad, depresión) y conductuales.

6. Entrenamiento en solución de problemas y en administración del tiempo. El entrenamiento en técnicas de solución de problemas puede reducir los conflictos que se generen en las relaciones familiares y sociales de las mujeres en esta etapa, sobre todo si se está viviendo de forma negativa y si se carece de los recursos necesarios para la solución acertada (Sueiro et al., 1999). Por eso, es importante aprender a llegar a acuerdos, y escoger la alternativa de respuesta más adecuada en cada situación para resolver dichos conflictos, reduciéndose así la ansiedad y/o depresión que generen estos, e incrementándose la calidad de vida y la autoestima de las mujeres. La administración del tiempo les ayuda a priorizar sus tareas y a manejar el estrés derivado de estas, así como a reservar parte del tiempo para actividades placenteras o de ocio.

La elección de todos estos componentes se ha basado en sus resultados positivos en otros programas de intervención, y su inclusión obedece al objetivo de diseñar un programa terapéutico que integre y abarque los diferentes aspectos que acontecen en la etapa menopáusica, ofreciendo alternativas, complementarias o exclusivas, al tratamiento farmacológico.

\section{RESULTADOS}

Previo al análisis de la eficacia de tratamiento se realizaron distintos análisis de homogeneidad, para comprobar que no hubiera diferencias entre los grupos en la fase de pretratamiento en las variables dependientes. Los resultados indicaron que no existían diferencias significativas entre los dos grupos. Asimismo se constató que la variable Terapeuta no interfirió en los resultados del estudio.

Se realizó un seguimiento a los 6 meses de haber concluido el programa de intervención a aquellas mujeres con las que se pudo contactar en la última fase de la investigación. Las muestras en el seguimiento fueron de 9 mujeres (grupo experimental) y 11 mujeres (grupo control). Dado que la muestra de ambos grupos se redujo en el seguimiento, se consideró necesario analizar si estos grupos eran equiparables a los iniciales. El análisis indicó que los dos grupos en seguimiento eran homogéneos a los grupos iniciales, antes y después del tratamiento, por lo que los resultados del seguimiento eran generalizables.

Los datos de la evaluación pretratamiento indican que:

(a) En el BMI, todas las mujeres del grupo experimental mostraban sintomatología física de moderada a alta. La media fue 
Tabla 3. Resumen de los contenidos del taller

\begin{tabular}{|c|c|c|}
\hline Sesión & Objetivos y contenido de la sesión & Técnicas \\
\hline \multirow{4}{*}{1} & Iniciar el programa de intervención psicológica en la menopausia. & Psicoeducación \\
\hline & Conocer, motivar e integrar a las participantes en este proceso. & \\
\hline & Enseñar los temas a desarrollar a lo largo del programa. & \\
\hline & Abordar los pensamientos y sentimientos que despierta el término menopausia. & \\
\hline \multirow[b]{2}{*}{2} & Explicar el aparato reproductor femenino y la función del ciclo hormonal. & Psicoeducación \\
\hline & $\begin{array}{l}\text { Determinar los cambios que se producen durante la menopausia a corto, medio y } \\
\text { largo plazo, y a diferentes niveles: fisiológico, físico y psicológico. }\end{array}$ & \\
\hline \multirow[t]{2}{*}{3} & $\begin{array}{l}\text { Explicar las distintas técnicas para tratar los cambios a corto plazo, indicando los } \\
\text { motivos que los señalan como convenientes. }\end{array}$ & Respiración \\
\hline & Explicar la relación tensión-ansiedad-malestar & Relajación \\
\hline 4 & $\begin{array}{l}\text { Explicar los ejercicios, postura y nutrición, para tratar los cambios a medio y largo } \\
\text { plazo. }\end{array}$ & $\begin{array}{l}\text { Entrenamiento en ejercicio } \\
\text { y composición de dietas }\end{array}$ \\
\hline \multirow{3}{*}{5} & $\begin{array}{l}\text { Explicar los trastornos de la musculatura del suelo pélvico en la postmenopausia y } \\
\text { promover los ejercicios de Kegel para su prevención y tratamiento. }\end{array}$ & Ejercicios de Kegel \\
\hline & $\begin{array}{l}\text { Transmitir información sobre las opciones existentes para combatir la sequedad va- } \\
\text { ginal (escozor, picor y dolor en las relaciones sexuales). }\end{array}$ & Reeducación sexual \\
\hline & Explicación de alternativas al coito. & \\
\hline
\end{tabular}

Explicar que son las Creencias Irracionales, que tipos de creencias son las más co- Reestructuración cognitimunes y mostrar en que momentos se presentan estas ideas.

Transmitir la forma de aprender a identificar los diferentes tipos de ideas irracionales.

Controlar pensamientos negativos.

Aprender a desmontar y modificar las ideas irracionales por pensamientos racionales.

Parada de pensamientos negativos recurrentes.

Parada de pensamiento

Aprender a identificar situaciones problemáticas en las que haya varias alternativas Solución de problemas de solución, facilitando posibles respuestas a la situación y establecer un procedi-

7 miento para escoger la respuesta conveniente al problema.

Explicar una de las formas para administrar el tiempo, identificando actividades Administración del tiemprioritarias, estableciendo objetivos y dividiéndolos en pasos para alcanzarlos. po

8

Evaluar la eficacia y grado de satisfacción del programa de intervención psicológica. Solución de dudas Cerrar el programa.

28,75 $(D T=5,75)$. Los síntomas más frecuentemente referidos, con una intensidad de moderada a alta, fueron los sofocos $(78,6 \%)$, el nerviosismo $(78,6 \%)$, la melancolía $(78,6 \%)$ y las mialgias $(71,4 \%)$. El grupo control mostró una puntuación media muy parecida $(M=28,88, D T=6,63)$. Los sofocos $(92 \%)$, la melancolía (68\%), el nerviosismo $(68 \%)$ y las mialgias $(56 \%)$ fueron los síntomas más frecuentes e intensos en este grupo. (b) En el HADS se registraron altas frecuencias de sintomatología ansiosa y/o depresiva intensa en ambos grupos. En la escala de ansiedad, el 96,1\% del grupo experimental y el $100 \%$ del grupo control puntuó por encima del criterio. El 39,3\% de las mujeres del grupo experimental y el $24 \%$ del grupo control presentaban sintomatología depresiva de moderada a alta.

(c) Respecto a calidad de vida, las mujeres del grupo experimental tenían un conocimien- 
to medio o bajo del climaterio, aunque presentaban una valoración negativa del mismo $(82,2 \%)$, presentaban sintomatología ansiosa y/o depresiva, percibían poco apoyo social y familiar, y el $57 \%$ de ellas informaba de problemas en su sexualidad y con la pareja. El 60\% de las mujeres del grupo control tenían un conocimiento medio acerca del proceso climatérico y el $84 \%$ lo valoraba negativamente. La mayoría presentaba sintomatología ansiosa y/o depresiva e informaban de poco apoyo familiar y problemas sexuales y de pareja.

Dado que las muestras de los grupos se redujeron sensiblemente, se decidió analizar los resultados también una prueba no parámetrica (que no imponen condiciones o supuestos tan restrictivos). Se utilizó para ello la prueba correspondiente, "prueba de los rangos con signo de Wilcoxon". Se obtuvieron los mismos resultados que con las pruebas paramétricas.

El análisis de los datos pre-postratamiento indicó que, en el grupo experimental, las puntuaciones bajaron en todas las variables una vez finalizada la intervención, lo que denota una mejoría estadística y clínicamente significativa en la sintomatología, tanto física como psíquica, y en la calidad de vida, como puede observarse en las Tablas 4 y 5.

La reducción de la sintomatología se mantuvo al cabo de 6 meses en el grupo experimental. Respecto al grupo control, no se registraron diferencias estadísticamente significativas en la fase postratamiento en ninguna VD; en el seguimiento se produjo un descenso en la ansiedad y en la puntuación global del BMI, aunque no se apreciaron diferencias estadísticamente significativas en ninguno de los síntomas por separado. Un resumen de los resultados se muestra en las Tablas 4 y 5 . Se incluyen los datos de pre-postratamiento en las tablas. Los resultados obtenidos en el postratamiento se analizan detalladamente en Larroy y Gutiérrez (2009). Aquí se hará referencia a los resultados obtenidos en el seguimiento.

Como puede apreciarse en la Tabla 4, existen diferencias significativas en las puntuaciones totales de todos los instrumentos empleados para la evaluación de las variables dependientes
(BMI, HADS y Cuestionario de Calidad de Vida), para el grupo experimental, entre los periodos de pretratamiento y seguimiento, así como en algunos de los síntomas más importantes, como los sofocos, la melancolía o las disestesias. Por otro lado, y con respecto al periodo de postratamiento, se observa que no existen apenas diferencias significativas, lo que indica que los resultados obtenidos en el postratamiento se mantienen al cabo de los seis meses de seguimiento.

La significación clínica de la intervención se evaluó en función del porcentaje de mujeres que alcanzaron puntuaciones indicativas de ausencia de trastorno o morbilidad. Se realizó, por tanto, un análisis sobre las puntuaciones obtenidas en el Índice de Kupperman y Blatt y en el HADS.

En relación con el pretratamiento, los resultados en el periodo de seguimiento fueron los siguientes:

(1) En el BMI, el 77,7\% de las mujeres del grupo experimental obtuvo en el seguimiento una puntuación inferior a 20 puntos (en el pretratamiento, ninguna mujer obtuvo una puntuación inferior a 20). Por el contrario, en el grupo control, ninguna de las mujeres alcanzó una puntuación menor de los 20 puntos en la situación de seguimiento

(2) En el grupo experimental se observa un descenso en el porcentaje de mujeres que presenta ansiedad de intensidad de moderada a alta; este porcentaje pasó del $96.1 \%$ inicial al 54\% tras seis meses de seguimiento. En el grupo control, el 100\% de las mujeres presentaba una ansiedad de moderada a alta en pretratamiento, $y$ en el seguimiento el porcentaje era del $90 \%$

(3) Asimismo, el 39,3\% de las mujeres del grupo experimental presentaba una depresión de moderada a alta en pretratamiento $\mathrm{y}$, el porcentaje se redujo al $25 \%$, a los 6 meses de seguimiento. En el grupo control, en el pretratamiento el $24 \%$ de las mujeres puntuó de forma moderada o alta y transcurridos 6 meses de seguimiento el porcentaje aumentó al 36,36\%. 
Tabla 4. $t$ de student y ANOVA de medidas repetidas: Variables dependientes en grupo experimental

\begin{tabular}{|c|c|c|c|c|c|c|}
\hline \multirow[b]{3}{*}{ Variables } & \multicolumn{6}{|c|}{ Grupo Clínico Experimental } \\
\hline & \multicolumn{2}{|c|}{$\begin{array}{c}\text { Pre-/Post-tratamiento } \\
\qquad(n=28)\end{array}$} & \multicolumn{2}{|c|}{$\begin{array}{l}\text { Pre-tratamiento/seguimiento } \\
\qquad(n=9)\end{array}$} & \multicolumn{2}{|c|}{$\begin{array}{c}\text { Post-tratamiento/seguimiento } \\
\qquad(n=9)\end{array}$} \\
\hline & $t_{(27)}$ & $F_{(1)}$ & $t_{(8)}$ & $F_{(1)}$ & $t_{(8)}$ & $F_{(1)}$ \\
\hline Ansiedad (HADS) & $6,11 * * *$ & & $6,06 * * *$ & & $2,47 *$ & \\
\hline Depresión (HADS) & $3,41 * * *$ & & $3,13 *$ & & 1,00 & \\
\hline $\begin{array}{l}\text { Índice Menopáusico } \\
\text { Kupperman y Blatt }\end{array}$ & $8,71 * * *$ & & $9,98 * * *$ & & 2,24 & \\
\hline Sofocos ${ }^{\mathrm{a}}$ & & $47,25 * * *$ & & $30,77 * * *$ & & 2,00 \\
\hline Parestesias $^{\mathrm{a}}$ & & 2,60 & & 2,28 & & 1,00 \\
\hline Insomnio $^{\mathrm{a}}$ & & $4,85^{\mathrm{b}}$ & & $8,00^{\mathrm{b}}$ & & 1,00 \\
\hline Nervios $^{\mathrm{a}}$ & & $7,56^{\mathrm{b}}$ & & 2,67 & & 2,29 \\
\hline Melancolía $^{a}$ & & $24,42 * * *$ & & $28,00 * * *$ & & 0.00 \\
\hline Vértigo $^{a}$ & & $6,24^{\mathrm{b}}$ & & 3,37 & & 0.00 \\
\hline Fatiga $^{a}$ & & 1,21 & & 2,67 & & $8,00^{\mathrm{b}}$ \\
\hline Mialgias $^{\mathrm{a}}$ & & $11,82 * *$ & & 1,56 & & 0.00 \\
\hline Cefaleas $^{\mathrm{a}}$ & & $5,23^{\mathrm{b}}$ & & 0,00 & & 2,29 \\
\hline Palpitaciones $^{\mathrm{a}}$ & & $4,81^{\mathrm{b}}$ & & $2,29^{\mathrm{b}}$ & & 2,29 \\
\hline Disestesias $^{\mathrm{a}}$ & & 0,81 & & $18,18 * *$ & & 2,70 \\
\hline $\begin{array}{l}\text { Cuestionario de Valora- } \\
\text { ción de Calidad de Vida }\end{array}$ & $3,88 * * *$ & & $6,78 * * *$ & & 1,92 & \\
\hline $\begin{array}{l}\text { Conocimiento } \\
\text { menopausia }^{\mathrm{a}}\end{array}$ & & $75,76^{* * *}$ & & $64,00 * * *$ & & 2,00 \\
\hline Valoración emocional $^{\mathrm{a}}$ & & 3,21 & & $10,00 * *$ & & 2,00 \\
\hline Ansiedad/Depresión ${ }^{a}$ & & $12,66^{* * *}$ & & 4,33 & & 1,22 \\
\hline Apoyo social $^{\mathrm{a}}$ & & $7,03^{\mathrm{b}}$ & & 0,18 & & 0,47 \\
\hline Apoyo familiar ${ }^{\mathrm{a}}$ & & $4,78^{\mathrm{b}}$ & & $8,61^{\mathrm{b}}$ & & 1,30 \\
\hline Sexualidad/ Pareja ${ }^{a}$ & & 0,52 & & $11,22 * *$ & & 1,00 \\
\hline
\end{tabular}

Nota.${ }^{a}$ Variables donde se aplicó corrección de Bonferroni. ${ }^{b}$ Variables es las que se obtuvo significación sin corrección de Bonferroni. HADS $=$ Hospital Anxiety and Depression Scale.

${ }^{*} p<0,05 ; * *<<0,01 ; * * p<0,001$.

En relación con el postratamiento, los resultados del grupo experimental en el periodo de seguimiento fueron los siguientes:

1. En el BMI, el porcentaje de mujeres con puntuación inferior a 20 pasó de $53,6 \%$ (postratamiento) al 77,7 \% (seguimiento). Es decir, se incrementa el porcentaje de mujeres con puntuaciones indicativas de ausencia de trastorno.
2, Respecto a la ansiedad, los resultados obtenidos en el postratamiento se mantienen en el seguimiento

3. Asimismo, se mantienen en el seguimiento los resultados obtenidos en el postratamiento

El tamaño del efecto conseguido por el programa de intervención en la reducción de la sintomatología se calculó mediante el programa G*Power (Buchner, Erdfelder y Faul, 1997). 
Tabla 5. $t$ de student y ANOVA de medidas repetidas: Variables dependientes en grupo control

\begin{tabular}{|c|c|c|c|c|c|c|}
\hline \multirow[b]{3}{*}{ Variables } & \multicolumn{6}{|c|}{ Grupo Control } \\
\hline & \multicolumn{2}{|c|}{$\begin{array}{l}\text { Pre-/Post-tratamiento } \\
\qquad(n=25)\end{array}$} & \multicolumn{2}{|c|}{$\begin{array}{l}\text { Pre-tratamiento/seguimiento } \\
\qquad(n=11)\end{array}$} & \multicolumn{2}{|c|}{$\begin{array}{l}\text { Post-tratamiento/seguimiento } \\
\qquad(n=11)\end{array}$} \\
\hline & $t_{(24)}$ & $F_{(1)}$ & $t_{(10)}$ & $F_{(1)}$ & $t_{(10)}$ & $F_{(1)}$ \\
\hline Ansiedad (HADS) & 1,77 & & $2,31 *$ & & 1,44 & \\
\hline Depresión (HADS) & $-0,84$ & & $-0,29$ & & 0,80 & \\
\hline $\begin{array}{l}\text { Índice Menopáusico } \\
\text { Kupperman y Blatt }\end{array}$ & 0,80 & & $3,15^{* *}$ & & 1,05 & \\
\hline Sofocos ${ }^{\mathrm{a}}$ & & 2,08 & & 0,00 & & 1,00 \\
\hline Parestesias $^{\mathrm{a}}$ & & 1,00 & & 1,00 & & 1,96 \\
\hline Insomnio $^{a}$ & & 0,14 & & 1,00 & & 2,22 \\
\hline Nervios $^{\mathrm{a}}$ & & 1,86 & & 5,71 & & 1,00 \\
\hline Melancolía $^{a}$ & & 0,27 & & 1,32 & & 1,00 \\
\hline Vértigo $^{a}$ & & 0,66 & & 1,00 & & 0,00 \\
\hline Fatiga $^{a}$ & & 0,00 & & 1,00 & & 1,00 \\
\hline Mialgias $^{\mathrm{a}}$ & & 0,00 & & 0,00 & & 0,00 \\
\hline Cefaleas $^{\mathrm{a}}$ & & 0,19 & & 2,22 & & 1,00 \\
\hline Palpitaciones ${ }^{\mathrm{a}}$ & & 0,19 & & 1,00 & & 0,00 \\
\hline Disestesias $^{\mathrm{a}}$ & & 0,06 & & 1,00 & & 0,18 \\
\hline $\begin{array}{l}\text { Cuestionario de Valora- } \\
\text { ción de Calidad de Vida }\end{array}$ & $-1,32$ & & $-1,04$ & & 0,41 & \\
\hline $\begin{array}{l}\text { Conocimiento } \\
\text { menopausia }^{\mathrm{a}}\end{array}$ & & 2,86 & & 2,22 & & 1,00 \\
\hline Valoración emocional $^{\mathrm{a}}$ & & 0,42 & & 0,37 & & 0,31 \\
\hline Ansiedad/Depresión ${ }^{\mathrm{a}}$ & & 0,56 & & 0,10 & & 0,07 \\
\hline Apoyo social ${ }^{\mathrm{a}}$ & & 3,52 & & 6,92 & & 0,37 \\
\hline Apoyo familiar ${ }^{\mathrm{a}}$ & & 3,85 & & 0,15 & & 8,33 \\
\hline Sexualidad/Pareja ${ }^{a}$ & & 0,27 & & 3,20 & & 0,64 \\
\hline
\end{tabular}

Nota. ${ }^{a}$ Variables donde se aplicó corrección de Bonferroni. HADS $=$ Hospital Anxiety and Depression Scale. ${ }^{*} p<0,05 ; * *<0,01$.

Tabla 6. Tamaño del efecto (TE) y potencia estadística para las variables dependientes

\begin{tabular}{lccccc}
\hline & \multicolumn{4}{c}{ Grupo Clínico Experimental } \\
\cline { 2 - 3 } & \multicolumn{2}{c}{$\begin{array}{c}\text { Pre-/Post-tratamiento } \\
(n=28)\end{array}$} & & $\begin{array}{c}\text { Pre-tratamiento/seguimiento } \\
(n=9)\end{array}$ \\
\cline { 2 - 3 } \cline { 5 - 6 } Variables & TE & Potencia & & TE & Potencia \\
\hline Ansiedad (HADS) & 1,17 & 0,99 & & 1,49 & 0,92 \\
Depresión (HADS) & 0,60 & 0,71 & & 0,77 & 0,47 \\
Índice Menopáusico Kupperman y Blatt & 1,28 & 0,99 & & 2,07 & 0,99 \\
Cuestionario de Valoración de Calidad de Vida & 0,63 & 0,75 & & 0,91 & 0,58 \\
\hline
\end{tabular}

Nota. HADS $=$ Hospital Anxiety and Depression Scale. 
En la Tabla 6 se refleja la potencia estadística y el tamaño del efecto conseguido por el tratamiento en cada una de las variables dependientes para el grupo experimental. Como puede apreciarse, se obtuvo un tamaño de efecto elevado para el BMI y la ansiedad, y medio para la calidad de vida y la depresión, aunque las potencias estadísticas para todas las variables fueron elevadas. En el seguimiento aumentó el tamaño del efecto para todas las variables.

Al finalizar el programa de intervención se entregó a todas las mujeres un autoinforme para conocer en qué medida el programa de intervención se había adecuado a sus necesidades y si, gracias a él, habían mejorado su situación respecto a la menopausia. El grupo clínico experimental obtuvo una puntuación media de 8 puntos en una escala de 0 a 10.

\section{DISCUSIÓN}

Aunque deben considerarse con cautela, debido a la reducción de los tamaños muestrales, los resultados obtenidos permiten constatar la eficacia a medio/largo plazo de la intervención aquí propuesta. Así, en la fase de seguimiento, el grupo experimental muestra una clara remisión de la sintomatología asociada a la menopausia (tanto física como psicológica) con respecto al pretratamiento. Las mujeres del grupo control, que no informaron cambios significativos en el postratamiento, tras un seguimiento de seis meses obtuvieron mejorías estadísticamente significativas en la puntuación total del Índice de Kupperman y Blatt y en la ansiedad según las puntuaciones del HADS, en relación con el pretratamiento. No obstante, las medias obtenidas por las mujeres de este grupo en ambas variables (27.64 en el Índice de Kupperman y Blatt y 9.09 en la escala de ansiedad del HADS) seguían alcanzando valores clínicos. Estos resultados pueden ser esperables, ya que la sintomatología asociada a la menopausia, en ausencia de tratamiento, puede remitir a largo plazo, por el simple transcurso del tiempo, pero el estudio aporta el dato de que esta remisión carece de significación clínica. Por el contrario, la intervención cognitivo-conductual logra la remi- sión estadística y clínicamente significativa de los síntomas en el postratamiento (Larroy y Gutiérrez, 2009) y una mayor reducción de los mismos en el seguimiento. Es decir, las mujeres sometidas a la intervención (frente a las del grupo control) obtuvieron mejores resultados, con datos indicativos de ausencia de trastorno y en menor intervalo de tiempo (de forma inmediata tras la intervención), resultados que se mostraron consistentes en el periodo de seguimiento.

Por lo que respecta a la variable Calidad de vida, también las mujeres del grupo experimental obtuvieron mejorías estadísticamente significativas, respecto a la línea base, mejorías que, incluso, superaban a las obtenidas en el postratamiento (Larroy y Gutiérrez, 2009). Por el contrario, ninguna de esas diferencias se manifiesta en el grupo control.

Analizando detalladamente cada variable se encuentra que en el grupo experimental se produjo un descenso en todos los síntomas del BMI, cambio especialmente significativo en los sofocos, la melancolía, las mialgias y las disestesias; en este último síntoma, la mejoría fue mayor que en el postratamiento (Larroy y Gutiérrez, 2009), aunque no alcanzó la significación estadística. Cabe destacar que, en el pretratamiento, las mujeres de este grupo puntuaron más alto en estos síntomas que en el resto de ítems del inventario.

Asimismo, tanto la ansiedad como la depresión remitieron de forma significativa en la muestra experimental, manteniéndose esta mejoría en la evaluación de seguimiento. El hecho de que las mujeres manifestaran no padecer ningún trastorno de tipo psicológico antes del proceso menopáusico, y que informaban de que sus niveles de ansiedad y depresión se habían incrementado al comienzo de éste, mejorando de forma significativa tras el tratamiento y continuando esta mejoría a largo plazo, denota que la intervención es adecuada para reducir los síntomas de depresión y ansiedad, que pueden ser frecuentes durante la menopausia.

Del mismo modo, la calidad de vida también mejoró de manera significativa en el grupo experimental en el seguimiento (más aún que en el postratamiento). La valoración emocional del proceso climatérico mejoró len- 
tamente, pues requirió de más tiempo para obtenerse diferencias estadísticamente significativas. Este cambio fue gradual y probablemente se empezó a producir cuando las mujeres automatizaron la puesta en práctica de las técnicas aprendidas durante la intervención, y percibieron cierto control sobre algunos de los síntomas. Tras la intervención, las mujeres del grupo experimental referían una mejoría en el apoyo social, familiar y en sus relaciones sexuales y de pareja (este cambio fue estadísticamente significativo al cabo de 6 meses). Por el contrario, el grupo control no informó de ningún cambio en su calidad de vida en el posttratamiento ni en el seguimiento. Esto indica la relevancia de realizar un seguimiento de todas las variables para conseguir una correcta evaluación del programa.

Tras estos resultados, puede afirmarse (con cautela) que el programa propuesto supone una buena alternativa de intervención a la THS, principalmente en aquellos casos en los que el tratamiento hormonal esté contraindicando; o, que incluso, puede suponer el tratamiento de elección, antes que la THS. Con respecto a esta última, la intervención cognitivo-conductual presenta las siguientes ventajas: carencia de efectos secundarios o indeseables; ahorro del gasto farmacéutico; ausencia de contraindicaciones; alta eficacia en la remisión de la sintomatología, remisión que se mantiene o incluso mejora a largo plazo; establece técnicas específicas para la sintomatología psicológica; e incluye profilaxis de trastornos que puedan desarrollarse a largo plazo. Así, por ejemplo, los componentes del programa de nutrición y ejercicio contribuyen a prevenir la osteoporosis, la obesidad y los trastornos cardiovasculares; los ejercicios de Kegel fortalecen el suelo pélvico, previniendo la aparición de pérdidas involuntarias de orina, y mejorando la actividad sexual, etc. Por otro lado, se trata de un protocolo de intervención sencillo, de fácil aplicación, de aplicación grupal (lo que mejora su eficiencia) y susceptible de ser utilizado en las diferentes instituciones dedicadas a la mujer.

Es deseable que una intervención además de ser eficaz y efectiva, sea eficiente (Labrador, Echeburúa y Becoña, 2000). La interven- ción presentada en este artículo se ha mostrado eficaz, puesto que ha conseguido la remisión, a medio/largo plazo, de la sintomatología asociada a la menopausia y la mejora de la calidad de vida en las mujeres climatéricas. Se ha realizado en un ambiente vinculado con la práctica habitual y con recursos muy reales, por lo que también ha sido efectiva. Y por último, ha supuesto un coste pequeño, en comparación con otras opciones terapéuticas, como las farmacológicas, por lo que es eficiente. Además, al realizarse de manera grupal, ha permitido que se aplicara a mayor número de mujeres en un tiempo relativamente corto. El formato grupal, además, proporciona un marco normativo de la sintomatología, favorece la expresión y la participación de las mujeres y colabora a la adherencia al tratamiento.

La posibilidad de contar con un grupo control ha sido otra ventaja de este trabajo, ventaja que en otros estudios no se ha contemplado y que ha permitido establecer que las mejorías obtenidas con la intervención no se debían únicamente al paso del tiempo.

Algo parecido ocurre con los terapeutas de programa; por razones ajenas a la investigación no se pudo aplicar la constancia o el balanceo como método para controlar esta variable. No obstante, se comprobó que este factor no interfirió en los resultados del tratamiento. Por el contrario, los resultados indican que este protocolo se ha aplicado con éxito por distintos terapeutas.

Un punto relevante de este trabajo se refiere a la intervención en las variables psicológicas asociadas a la menopausia: ansiedad y depresión. Las elevadas puntuaciones alcanzadas en los ítems del nerviosismo y la melancolía del BMI en la situación pretratamiento, son congruentes con los datos obtenidos en ambas escalas del HADS. Estos datos indican que la sintomatología psicológica es muy importante en el proceso menopáusico. Por este motivo, las intervenciones deben contemplar estrategias para paliar tanto síntomas psicológicos como fisiológicos. El hecho de que coexistan ambos en el proceso menopáusico hace sospechar una especie de "círculo vicioso". Así, la sintomatología física exacer- 
ba la aparición de la sintomatología psicológica (por ejemplo, la repetición continuada de sofocos provoca malestar o ansiedad en la mujer y fatiga, por el insomnio asociado, 1legando a limitar su vida cotidiana, lo que puede llegar a provocar disforia y depresión); y a la inversa, los síntomas psicológicos precipitan los síntomas físicos (situaciones que generen estrés o ansiedad en la mujer predisponen la aparición de sofocos y de insomnio). En este sentido, y, con vistas a mejorar el programa, sería conveniente, en futuros trabajos, incidir más en la intervención de ansiedad y depresión, mediante, por ejemplo, la inclusión de técnicas netamente conductuales (programas de reforzamiento, actividades placenteras) y, probablemente, de técnicas de mejora de la autoestima.

Cabe destacar el gran desconocimiento acerca del proceso menopáusico informado por las mujeres, que, de forma mayoritaria, valoraban este proceso como algo negativo al inicio de la intervención; aún desconociendo cuáles eran las posibles consecuencias de este nuevo acontecimiento en sus vidas, prejuzgaban que iba a ser altamente perjudicial para ellas. Es muy probable que la falta de información fuera responsable de esta valoración, y, en este sentido, el papel de la psicoeducación ha sido fundamental.

Las intervenciones durante esta etapa, además de aliviar la sintomatología, deben fomentar una mejora en la calidad de vida de las mujeres. Ésta puede verse muy afectada, por lo que es importante trabajar este constructo (Galán, Pérez, Martín y Bordás, 2008). Los cambios producidos por la intervención en este ámbito se han manifestado a medio plazo, pues, como se comentó anteriormente, el proceso menopáusico puede abarcar muchos meses, de ahí la importancia de insistir en el correcto aprendizaje de las diferentes técnicas contempladas en el tratamiento y realizar medidas de seguimiento para evaluar el correcto funcionamiento de la intervención. En este sentido, un seguimiento más largo es deseable y se abordará en futuras investigaciones. Asimismo, el reducido número de mujeres que se pudo evaluar en el seguimiento hace que los datos de este estudio deban contemplarse con cautela.
Parece claro que ambos factores (la duración del seguimiento y el reducido número de mujeres en las muestras en este periodo) han sido las limitaciones más importantes de este estudio. Sin embargo, la significación estadística alcanzada en el cambio de la mayoría de las variables dependientes anima a la réplica del trabajo con muestras mayores y con periodos de seguimiento más largos.

En resumen, ha quedado demostrado que una intervención cognitivo-conductual es eficaz en la remisión de sintomatología menopáusica. Y es importante destacar que los cambios se mantienen y se incrementan tras el seguimiento, más allá de la acción del paso del tiempo. Es muy probable que la explicación de que los cambios a medio/largo plazo sean mayores que a corto plazo resida, precisamente, en la habilidad, progresivamente creciente, de las mujeres para poner en marcha y automatizar las estrategias adquiridas durante la intervención. Es, por tanto, esperable, y puede ser objeto de futuras investigaciones, que los cambios se mantengan e incrementen en periodos de seguimiento mayores. Por tanto, cuanto antes se intervenga en las mujeres con sintomatología menopáusica más beneficios obtendrán éstas.

\section{REFERENCIAS}

Anderson, D., Yoshizawa, T., Gollschewski, S., Atogami, F., y Courtney, M. (2004). Relationship between menopausal symptoms and menopausal status in Australian and Japanese women. Nursing and Health Sciences, 6, 173-180

Barczak, P., Kane, N., y Andrews, S. (1988). Patterns of psychiatric morbidity in a genito-urinary clinic. British Journal of Psychiatry, 152, 698-700.

Buchner, A., Erdfelder, E., y Faul, F. (1997). How to Use G*Power. Disponible en (02/03/2006): http://www. psychologie.uni-trier.de:8000/projects/gpower/ howtousegpower.html.

Camuñas, N., García, E., Vivas, F., Morales, C., Aranda, D., y Cano, A. (2001). Intervención psicológica en mujeres menopáusicas con hipertensión. Psicología. com, 5. Documento disponible en (01/02/2006): http://www.psiquiatria.com/psicologia/revista/ $62 / 2439 / ?++$ interactivo

De la Gándara-Martín, J.J. (1997). Menopausia y Trastornos psicosomáticos. Burgos: Cauces de Opinión. 
De las Cuevas, C., García-Estrada, A., y González, J.L. (1995). "Hospital Anxiety and Depresión Scale" y Psicopatología Afectiva. Anuales de Psiquiatría, 2, 126-130.

Fernández, E., Magnani, E, Fernández, J.M., y Fernández-Arcilla (2000). Menopausia ¿Qué es? Madrid: Lilly.

Freedman, B.R., y Woodward, S. (1992). Behavioral treatment of menopausal hot flushes: evaluation by ambulatory monitoring. American journal of obstetrics and gynecology, 167, 436-439.

Fu, S., Anderson, D., y Courtney, M. (2003). Cross-cultural menopausal experience. Comparison of Australian and Taiwanese women. Nursing and Health Sciences, 5, 77-84.

Galán, A., Pérez, A., Martín, A., y Bordás, M. (2008). Calidad de vida de los trasplantados en relación a otras situaciones médicas estresantes vividas por los enfermos pulmonares. Psicothema, 20, 266-272.

Germaine, L.M., y Freedman, R.R. (1984). Behavioural treatment of menopausal hot flashes: Evaluation by objective methods. Journal of Consulting and Clinical Psychology, 52, 1072-1079.

Hernández, J., De la Gándara-Martín, J., y Sánchez, M.A. (2003). Utilidad de la terapia hormonal sustitutiva en los trastornos en los trastornos depresivos durante la menopausia. Estado de cuestión. Anuales de Psiquiatría, 19, 172-178.

Hunter, M.S. (2003). Cognitive behavioural interventions for premenstrual and menopausal symptoms. Journal of reproductive and Infant Psychology, 21, 183-193.

Hunter, M.S., y Liao, K.L.M. (1996). Evaluation of a four session cognitive-behavioural intervention for menopausal hot flushes. British Journal of Health Psychology, 1, 113-125.

Keefer, L.A. (2003). The effect of a cognitive-bahavioral group treatment on perimenopausal hot flashes and related symptoms. Dissertation Abstracts international: Section B: The Sciences and Engineering, 64(6B), 2923.

Kupperman, H. S., y Blatt M. H. (1953). Comparative clinical evaluation of estrogen preparations by menopausal and amenorreheal indices. Journal of Clinical Endocrinology, 13, 688-703.

Labrador, F.J., Echeburúa, E., y Becoña, E. (2000). Guía para la elección de tratamientos psicológicos efectivos. Hacia una nueva psicología clínica. Madrid: Dykinson-Psicología.

Larroy, C. (2004). Trastornos especificos de la Mujer. Madrid: Síntesis.

Larroy, C., y Gutiérrez, S. (2009). Intervención cognitivoconductual en sintomatología menopáusica: efectos a corto plazo. Psicothema, 21, 260-266.

Larroy, C., Gutiérrez, S., y León, L. (2004). Tratamiento cognitivo-conductual de la sintomatología asociada a la menopausia. Avances en Psicología Latinoamericana. Psicología de la Salud de la Mujer. 22, 77-88.

Magnani, E., Sánchez-Arcilla, I., Aranda, D., Campillo, F., Campillos, M., de Dios, M.I., García, P., García, P., y Rodríguez, A. (1999). Programa de Menopausia: Un buen momento para pensar en sí mima. Ayuntamiento de Madrid Área de Salud y Consumo. Madrid: Ediciones Díaz Santos.

Marín, R.M. (1996). Menopausia y Salud. Atención Integral a la Mujer de Mediana Edad. En J. Sánchez-Cánovas, Menopausia y Salud. Barcelona: Ariel.

Marín, R.M., Sánchez-Cánovas, J., y Dupuy, M.J. (1995). Assessing the effects of Health Promotion Programs on subjective Well-being of menopausal women. En Rodriguez-Marín (Ed.), Health Psychology and Quality of life Research. Proceedings of the $8^{\text {th }}$ annual conference of the European Health Psychology Society, 2, 906-915.

Matthews, K.A. (1991). Myths and realities of the menopause. Annual meeting of the American Psychosomatic Society. Santa Fe, Nuevo México. Psychosomatic Medicine, 54, 1-9.

Moorey, S., Greer S., Watson, M., y Gorman, C. (1991). The factor structure and factor stability of the hospital anxiety and depression scale in patients with cancer. British Journal of Psychiatry, 158, 255-259.

Morrison, M.F., Kallan, M J., Have, T.T., Katz, I., Tweedy, K., y Battistini, M. (2004). Lack of efficacy of estradiol for depression in postmenopausal women. A randomized, controlled trial. Biological Psychiatry, 55, 406-412.

OMS (1996). Investigaciones sobre la menopausia en los años noventa: Informe de un grupo cientifico de la OMS. Ginebra: OMS (Serie de informes técnicos $\mathrm{n}^{\circ}$ 866).

Palacios, S., Menéndez, C., Jurado, A.R., y Castaño, R. (1995), Changes in sex behavior after menopause: effects of tibolone. Maturitas, 22, 155-161.

Pelcastre-Villafuerte, B., Garrido-Latorre, M.C., y de León-Reyes, V. (2001). Menopausia. Representaciones sociales y prácticas. Salud pública de México, 43, 408414.

Philips, H.C. (1991) El tratamiento psicológico del dolor crónico. Madrid: Pirámide (or., 1988)

Rosario, E.J., Villani, R.G. Harris, J., y Klein, R. (2003). Comparison of Strength training Adaptations in Early and Older Postmenopausal Women. Journal of aging and physical activity, 11, 143-155.

Salvatierra, V. (1992). Alteraciones psicológicas y sexuales durante la menopausia. En S. Palacios, Climaterio y Menopausia. Madrid: Mirpal.

Sánchez-Cánovas, J. (1999). Cuestionario de valoración de la calidad de vida, en mujeres de 45 a 64 años. Madrid: Ministerio de Asuntos Sociales, Instituto de la Mujer. 
Sociedad Española de Ginecología y Obstetricia (2006). La Menopausia en España. Calidad de Vida y Tratamientos. Madrid: EPES.

Stevenson, D.W., y Delprato, D.J. (1983). Multiple component self-control program for menopausal hot flashes. Journal of Behaviour Therapy and Experimental Psychiatry, 10, 137-140.
Sueiro, E., Carbulleira, M.M., Perdiz, C., Rodríguez, J.A., y González, A. (1999). Promoción de la salud. Intervención psicológica en un grupo de mujeres en climaterio. Una experiencia práctica. Psicología conductual, 7, 155-167. Zigmond, A.S., y Snaith, R.P. (1983). Hospital Anxiety and Depression Scale. Acta Psychiatric Scandinavian, 67, 361-370. 